\title{
Pseudogap Behaviour in FeTe and FeSe Probed By Photoemission
}

\author{
P. Mishra ${ }^{1, *}$, H. Lohani ${ }^{1}$, R.A. Zargar ${ }^{2}$, V.P.S. Awana ${ }^{3}$ and B. R. Sekhar ${ }^{1}$ \\ ${ }^{1}$ Institute of Physics, Sachivalaya Marg, Bhubaneswar-751005, India \\ ${ }^{2}$ Department of Physics, Jamia Millia Islamia, New Delhi-110025, India \\ ${ }^{3}$ CSIR-National Physical Laboratory, Dr. K. S. Krishnan Marg, New Delhi-110012, India \\ "E-mail:pramitam@iopb.res.in
}

\begin{abstract}
This study reports the valence band photoelectron spectroscopic studies of FeTe and FeSe. A doping induced and temperature dependent pseudogap occur near the Fermi level. The spectral weight transfer occurs due to the change in chalcogen height due to lowering of temperature. This result is in analogy with the reduction in chalcogen height due to the replacement of Te by Se.
\end{abstract}

Keywords: UV photoemission spectroscopy, superconductor, electronic structure, pseudogap regime

PACS: 74.25.Jb, 74.70.Xa, 74.20. Pq, 74.72.Kf

\section{INTRODUCTION}

Discovery of superconductivity in iron based compounds[1,2], has led to an upsurge of research in the field of high Tc superconductors due to their fundamental and technological importance. Among the iron superconductors, $\mathrm{Fe}(\mathrm{Te}, \mathrm{Se})$ compounds are found to be showing the strongest electron correlation[3]. Although $\mathrm{Fe}(\mathrm{Se}, \mathrm{Te})$ is rather simple from a structural point of view, it shows a strong bearing of the spin fluctuations and structural and magnetic disorder on its superconducting properties. These compounds crystallize in a tetragonal crystal structure with a simple stacking of edge sharing $\mathrm{Fe}(\mathrm{Se}, \mathrm{Te})_{4}$ tetrahedra[2].

Recent photoemission studies on $\mathrm{Fe}(\mathrm{Te}, \mathrm{Se})$ systems have shown that the electronic properties of these systems are governed by the $\mathrm{Fe} 3 \mathrm{~d}$ and chalcogen $2 p$ states near Fermi Level $\left(E_{F}\right)[4,5]$. Changes in the Fe3d - Se2p correlation strength with doping or temperature result in noticeable spectral weight shifts in the near $\mathrm{E}_{\mathrm{F}}$ states. Earlier studies[6,7] have shown that these near $E_{F}$ spectral weight shifts lead to the formation of a pseudogap which intimately related to the superconducting properties of these materials, although the origin and nature of the pseudogap are still under debate.

In this study, we have studied the temperature dependent normal state pseudogap behavior using angle integrated valence band photoelectron spectroscopy. An analogy between doping induced pseudogap and temperature induced pseudogap is discussed.

\section{EXPERIMENTAL}

Polycrystalline samples of $\mathrm{FeSe}_{1-\mathrm{x}} \mathrm{Te}_{\mathrm{x}}(\mathrm{x}=0,1)$ and FeSe were synthesized via solid state reaction route [8] and characterized using XRD and resistivity measurements[9]. Angle integrated ultraviolet photoemission measurements were performed at $300 \mathrm{~K}$ and $77 \mathrm{~K}$ using high intensity VUV source (He I (21.2 $\mathrm{eV})$ and $\mathrm{He}$ II $(40.8 \mathrm{eV}))$ and a hemispherical electron energy analyser (SCIENTA R3000). Fermi energies were calibrated using a freshly evaporated Ag film on sample holder. The total energy resolution estimated from the width of the Fermi edge, was about $27 \mathrm{meV}$ for He I excitation. All photoemission measurements were performed at a base vacuum of $5.0 \times 10^{-11} \mathrm{mbar}$ after scraping the sample surfaces in situ. For the temperature dependent measurements, the samples were cooled using liquid nitrogen.

\section{RESULTS AND DISCUSSIONS}

Figure 1 shows the He I valence band spectra of $\mathrm{FeTe}$ and $\mathrm{FeSe}$. The spectral features marked $\mathrm{A}$ at 0.5 $\mathrm{eV}$ and $\mathrm{B}$ at $2 \mathrm{eV}$, correspond to $\mathrm{Fe} 3 \mathrm{~d}$ states. Peak C 
at $4 \mathrm{eV}$ is related to the hybridized $\mathrm{Fe} 3 \mathrm{~d}-\mathrm{Se} 4 \mathrm{p} / \mathrm{Te} 5 \mathrm{p}$ states while peak D at $6 \mathrm{eV}$ correspond to $\mathrm{Se} 4 \mathrm{p} / \mathrm{Te}$ $5 \mathrm{p}$ states. The marked experimental features match with the earlier theoretical calculations $[3,10]$ and experimental results of Yokoya et al [5].

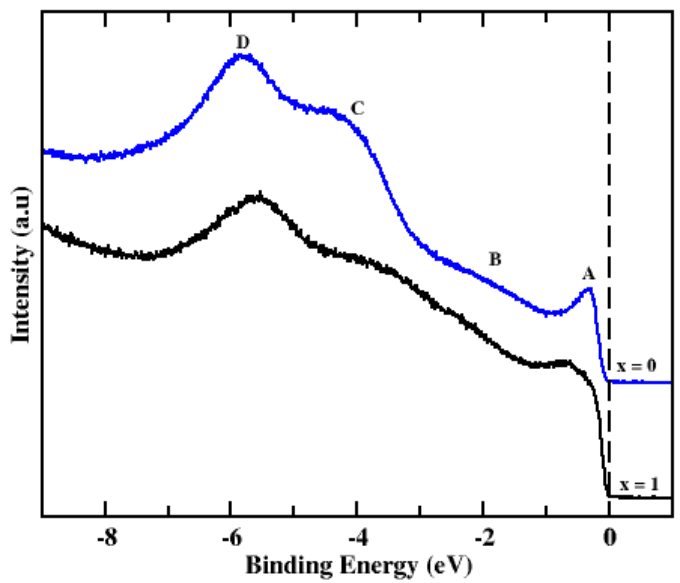

Figure 1. Valence band spectra taken using He I for $\mathrm{FeTe}(\mathrm{x}=1)$ and FeSe $(\mathrm{x}=0)$. The dotted line represents the Fermi level.

All the features show an evolution in its spectral shape as a function of doping. The broad feature A, in case of FeTe transforms in to a prominent peak in case of FeSe. Features B and C which are overlapping in case of FeTe, becomes well separated for FeSe. The broad feature $\mathrm{C}$ becomes prominent and shifts to higher binding energy with Se inclusion. Peak D also shifts to higher binding energy with increase in Se content.

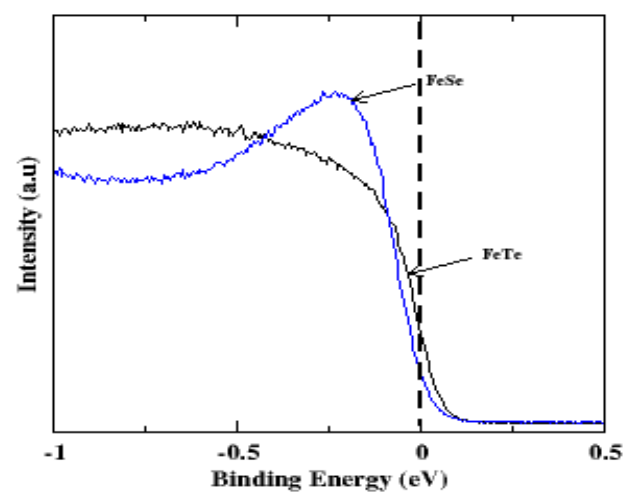

Figure 2. Near $\mathrm{E}_{\mathrm{F}}$ valence band spectra for FeTe and FeSe taken at $300 \mathrm{~K}$ using He I.

The room temperature near $\mathrm{E}_{\mathrm{F}}$ spectra (fig 2) shows the spectral evolution as a function of doping. Replacement of Se by Te leads to spectral weight depletion at Fermi level with a simultaneous enhancement in intensity at higher binding energy. Similar doping dependent pseudogap has also been observed by Yokoya et al. [5].

The high resolution near $\mathrm{E}_{\mathrm{F}}$ spectra (till $0.8 \mathrm{eV}$ ) taken at $300 \mathrm{~K}$ and $77 \mathrm{~K}$ for both the compositions shown in fig 2, clearly reveal the doping dependence of the near $\mathrm{E}_{\mathrm{F}}$ features. The doublet structure (shown by black bar in fig 2) in case of FeTe evolves into a prominent peak in case of FeSe. The observed change reflects the change in the distance of chalcogen atom from $\mathrm{Fe}$ plane. Replacement of Te by Se results in reduction of Fe chalcogen height along with an increase of chalcogen - Fe - chalcogen bond angle[11].

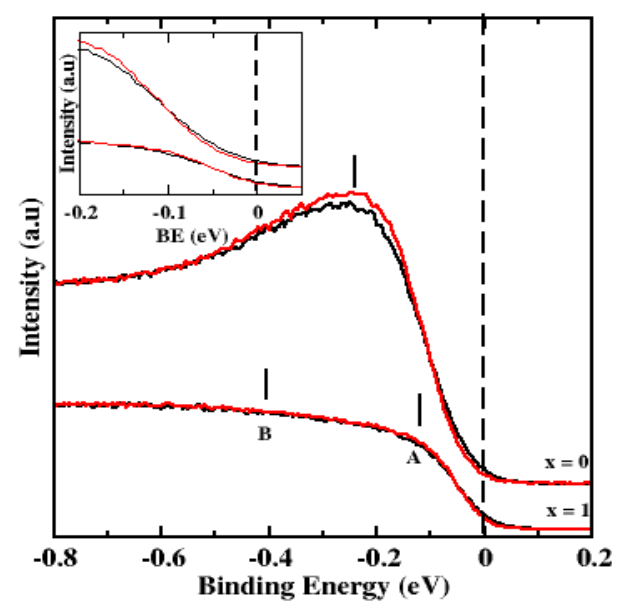

Figure 3. High resolution near $\mathrm{E}_{\mathrm{F}}$ spectra taken at 300 $\mathrm{K}$ (black) and $77 \mathrm{~K}$ (red) for FeTe $(\mathrm{x}=1)$ and $\mathrm{FeSe}(\mathrm{x}=0)$. Inset shows the zoomed picture near Fermi level.

As temperature is lowered, there occurs a depletion of density of states near $\mathrm{E}_{\mathrm{F}}$ and a corresponding enhancement in density of states at higher binding energy at position A. This temperature dependent change in the spectral weight is more prominent in the case of FeSe than FeTe (inset fig 2). The area by which the A peak has increased does not match with the number of states depleted from the near $E_{F}$ position. This indicates that electrons from other orbitals also shift resulting in the increase in spectral weight at A. The depletion of these DOS clearly indicates the opening of a pseudogap with lowering of temperature.

Comparison of fig 2 and 3 shows that the there is an analogy between the doping dependent pseudogap occuring at $300 \mathrm{~K}$ and temperature dependent pseudogap. The doping dependent pseudogap arises due to the changes in the chalcogen height from the $\mathrm{Fe}$ plane. According to Horigane et al. [12], lowering of temperature leads to a reduction in the chalcogen height from the Fe plane. This suggests that the 
pseudogap arising as a result of lowering in temperature is also an effect of change in Fe chalcogen height which is reflected in the spectral weight depletion at Fermi level. The magnitude of pseudogap is greater in case of FeSe as the reduction in chalcogen height at low temperature for $\mathrm{FeSe}$ is more in comparison to FeTe. The lower chalcogen height in case of FeSe leads to greater correlation and hence shows a clear pseudogap than FeTe. The experimental results observed are all above the Neel temperature (70 $\mathrm{K})$ in case of FeTe and superconducting transition temperature $(8 \mathrm{~K})$ in case of FeSe. Hence, this pseudogap is not linked to the superconducting gap or polaronic effects. The formation of pseudogap shows the electron correlation effects in the family of $\mathrm{Fe}(\mathrm{Se}, \mathrm{Te})$ superconductors.

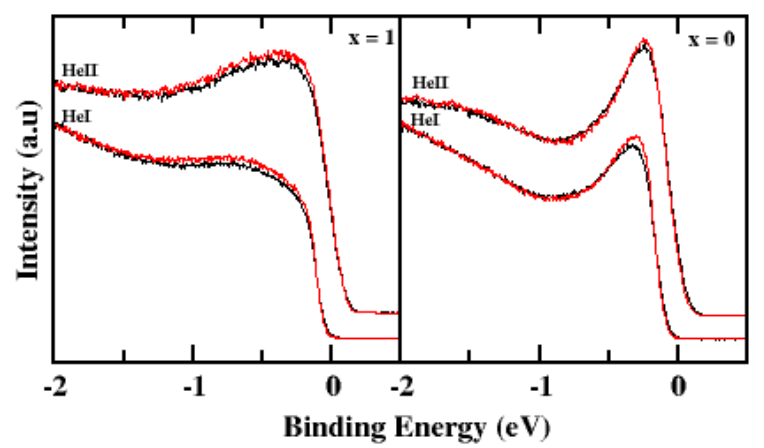

Figure 4. Comparison of He I and He II spectra taken at $300 \mathrm{~K}$ (black) and $77 \mathrm{~K}$ (red) for FeTe and FeSe

Fig 4. shows the spectra collected using He II $(40.8 \mathrm{eV})$ photons at $300 \mathrm{~K}$ and $77 \mathrm{~K}$ for FeTe and FeSe. The Fe $3 \mathrm{~d}$ derived density of states is greater in case of He II than He I due to the higher cross section of $\mathrm{Fe} 3 \mathrm{~d}$ at $\mathrm{He}$ II photon energy. The spectral weight shifts and thereby arising pseudogap, follow the same trend in case of He II also but with a lower magnitude compared to $\mathrm{He} \mathrm{I}$.

In conclusion, we have studied the temperature dependent and doping dependent spectral weight changes in FeTe and FeSe. The change in the structural topology leads to the formation of pseudogap in case of $\mathrm{Fe}(\mathrm{Se}, \mathrm{Te})$.

\section{REFERENCES}

1. Y. Kamihara, H. Hiramatsu , M. Hirano, R. Kawamura, H. Yanagi, T. Kamiya and H. Hosono, J. Am. Chem. Soc. 128(31), 10012 (2006).

2. K.-W. Yeh, T.-W. Huang, Y.L. Huang, T.-K. Chen, F.-C. Hsu, P.M. Wu, Y.-Y. Lee, Y.-Y. Chu, C.-
L. Chen, J. -Y. Luo, D.-C. Yan and M.-K. Wu, Europhys. Lett. 84, 37002 (2008).

3. T. Miyake, K. Nakamura, R. Arita and M. Imada, $J$. Phys. Soc. Jpn. 79, 044705 (2010).

4. R. Yoshida, T. Wakita, H. Okazaki, Y. Mizuguchi, S. Tsuda, Y. Takano, H. Takeya, K. Hirata, T. Muro, M. Okawa, K. Ishizaka, S. Shin, H. Harima, M. Hirai, Y. Muraoka and T. Yokoya, J. Phys.

Soc. Jpn. 78, 034708 (2009).

5. T. Yokoya, R. Yoshida, Y. Utsumi, K. Tsubota, H. Okazaki, T. Wakita, Y. Mizuguchi, Y. Takano, T. Muro, Y. Kato, H. Kumigashira, M. Oshima, H. Harima, Aiura Y, Sato H, Ino A, Namatame

H, Taniguchi M, Hirai M, Muraoka Y, Sci. Technol. Adv. Mater. 13, 054403 (2012).

6. Y. Zhang, F. Chen, C. He, L.X. Yang, B.P. Xie, Y.L. Xie, X.H. Chen, M. Fang, M. Arita, K. Shimada, H. Namatame, M. Taniguchi, J.P. Hu and D.L. Feng, Phys. Rev. B 82, 165113 (2010).

7. K. Okazaki, Y. Ito, Y. Ota, Y. Kotani, T. Shimojima , T. Kiss, S. Watanabe, C, -T Chen, S. Niitaka, T. Hanaguri, H. Takagi, A. Chainani and S. Shin, Scientific Reports 4, 4109 (2014).

8. V.P.S. Awana, A. Pal, A. Vajpayee, M. Mudgel, H. Kishan, M. Husain, R. Zeng, S. Yu, Y. F. Guo, Y. G. Shi, K. Yamaura and E.T. -Muromachi, J. Appl. Phys. 107, 09E128 (2010).

9. R. A. Zargar, A. Pal, A.K. Hafiz, V.P.S. Awana, J. Supercond. Nov. Magn. 27, 897 (2014).

10. A. Subedi, L. Zhang, D.J. Singh and M.H. Du, Phys. Rev. B 78, 134514 (2008).

11. Z.P. Yin, K. Haule and G. Kotliar, Nature Materials. 10, 932 (2011).

12. K. Horigane, H. Hiraka and K. Ohoyama $2009 \mathrm{~J}$. Phys. Soc. Jpn. 78, 074718 (2009). 
AIP Conference Proceedings is copyrighted by AIP Publishing LLC (AIP). Reuse of AIP content is subject to the terms at: http://scitation.aip.org/termsconditions. For more information, see http://publishing.aip.org/authors/rights-and-permissions. 